\title{
Perspective
}

PERSPECTIVE Actualité en histoire de l'art

$2 \mid 2013$

\section{Le Brésil}

\section{Fabrique et promotion de la brésilianité : art et enjeux nationaux}

creating and promoting Brazilianness: art and national implications

\section{Jorge Coli}

Traducteur : Cécile Lombard

\section{(2) OpenEdition \\ Journals}

Édition électronique

URL : http://journals.openedition.org/perspective/3865

DOI : $10.4000 /$ perspective.3865

ISSN : 2269-7721

\section{Éditeur}

Institut national d'histoire de l'art

\section{Édition imprimée}

Date de publication : 31 décembre 2013

Pagination : 213-223

ISSN : 1777-7852

Référence électronique

Jorge Coli, «Fabrique et promotion de la brésilianité : art et enjeux nationaux », Perspective [En ligne],

2 | 2013, mis en ligne le 30 juin 2015, consulté le 01 octobre 2020. URL : http://

journals.openedition.org/perspective/3865; DOI : https://doi.org/10.4000/perspective.3865 


\section{Débat}

\section{Travaux}

Actualité

\section{Fabrique et promotion de la brésilianité : art et enjeux nationaux}

Jorge Coli

Première constatation : la production artistique du Brésil, depuis le début de son histoire, n'a jamais été fondée sur l'observation. Tant dans les arts plastiques qu'en littérature, artistes, intellectuels et écrivains, aveugles à la réalité de ce qui les entourait, se sont enfermés dans un monde imaginaire.

Cette remarque liminaire, simple et générique annonce les caractéristiques fondamentales de l'art brésilien ; cette spécificité est issue de croyances idéologiques, et en particulier de la notion d'identité nationale et de racines. Ces fictions, ces constructions de l'esprit, ces fabulations sont bien ancrées dans les perceptions et les attentes des individus, comme celles des groupes humains, dans les comportements individuels et collectifs, les formes de pensée et la conception du monde. En ce qui concerne l'histoire des arts, une forte tendance au nationalisme a également de tout temps remplacé l'examen et l'analyse approfondie de son objet.

Par conséquent, si nous considérons deux catégories de production artistique, celle construite à partir de l'imaginaire et celle issue de l'observation, nous pouvons avancer que, dans la culture du Brésil, la première est infiniment plus forte.

Le bref épisode de l'occupation néerlandaise du Nordeste au XVII ${ }^{e}$ siècle ${ }^{1}$, au temps où le Brésil était une colonie portugaise, de 1500 à 1822, nous fournit un élément de comparaison : les œuvres d'art exécutées au Brésil par les Néerlandais s'appuyaient sur l'observation immédiate, in praesentia ; c'était l'une des plus riches particularités de ces artistes protestants, modernes, à l'esprit scientifique. Frans Post, par exemple, même lorsqu'il peignait le Brésil de mémoire après son retour aux Pays-Bas, a toujours fourni un témoignage de la réalité (fig. 1). Sur les trois siècles pendant lesquels le pays fut une colonie portugaise, la plupart des documents historiques visuels relatant la vie quotidienne $d^{\prime}$ alors datent des huit ans pendant lesquels Maurice de Nassau a gouverné le Nordeste.

A morte do Padre Filipe Bourel, un tableau singulier et l'un des rares exemples de peinture coloniale à échapper aux thèmes religieux canoniques, offre un contraste évident avec cette production hollandaise " réaliste » (XVIII siècle, Rio de Janeiro, Museu Nacional de Belas Artes ; fig. 2). Le catalogue du musée l'attribue
Après avoir suivi une partie de son parcours en France (formation et enseignement dans des universités françaises, collaborateur du journal Le Monde, etc.) et au Brésil, Jorge Coli est depuis les années 1990 professeur et directeur de l'institut de philosophie et des sciences humaines à I'Universidade Estadual de Campinas. Il fut invité dans plusieurs universités, comme celles de Princeton et d'Osaka. Ses recherches portent notamment sur l'art du XIX ${ }^{\mathrm{e}}$ siècle brésilien et français, et il a, entre autres, publié Música

final: Mário de Andrade

e sua coluna jornalística

Mundo musical (1998), L'Atelier de Courbet (2007),

O corpo da liberdade: reflexões sobre a pintura do século XIX (2010). 


\section{LE BRÉSIL}

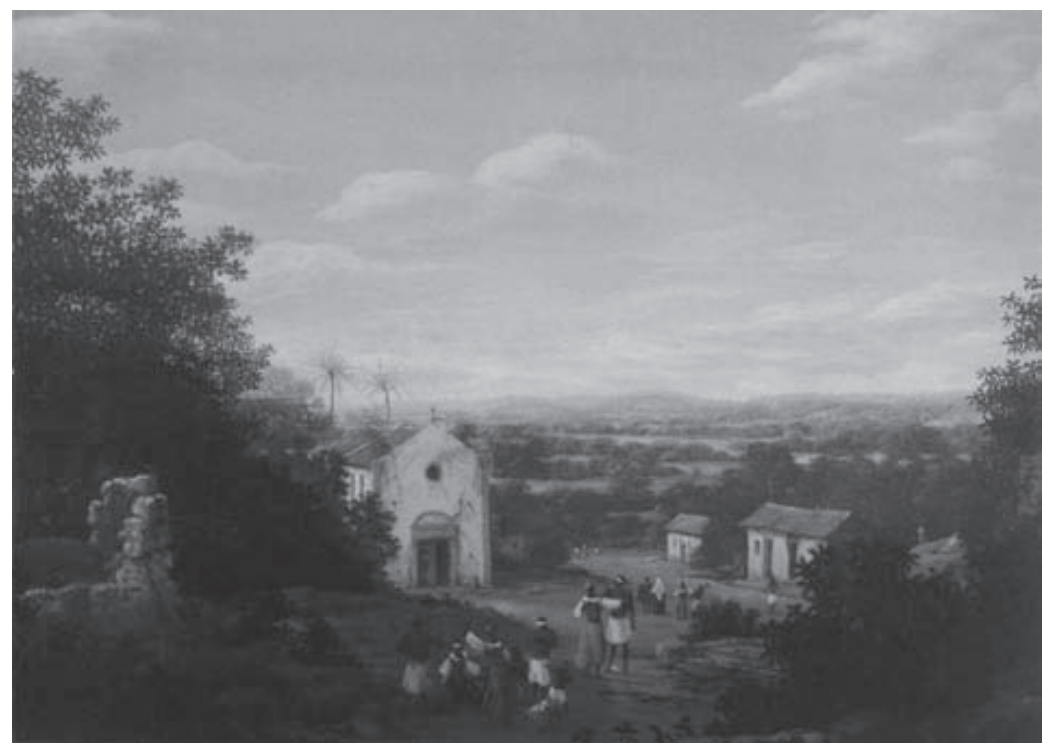

à un auteur inconnu de $I^{\prime}$ "École portugaise »du XVIII ${ }^{e}$ siècle $^{2}$, et certaines hypothèses le situent aux alentours de 1730. Le déchiffrage iconographique en est fascinant ${ }^{3}$. La localisation sur une colline près de deux cités fortifiées (dont il n'existe aucun équivalent dans I'architecture du pays) et les palmiers exotiques clairement identifiables comme des dattiers - espèce nonendémique au Brésil mais chargée en résonances symboliques intenses (arbre du Paradis, fuite en Égypte, métaphore de la croix); le perroquet, oiseau exotique qui est certes une réalité abondante au Brésil, mais qui reprend surtout l'image de la parole missionnaire

1. Frans Post, Igreja de São Cosme e Damião em Igarassu, vers 1637-1645, Rio de Janeiro, Museu Nacional de Belas Artes.
2. Anonyme, Morte do Padre Filipe Bourel, vers 1730, Rio de Janeiro, Museu Nacional de Belas Artes. utilisée, par exemple, par le père Andrea Pozzo au plafond de l'église Saint-Ignacede-Loyola à Rome pour illustrer l'Allégorie du travail missionnaire des Jésuites ${ }^{4}$; la cahute, refuge précaire du missionnaire à l'agonie, abri de bois dont la fragilité s'oppose à la solidité des cités aperçues au loin, dévoile les principes de la culture érudite et humaniste sur lesquels repose le tableau. Pour le peintre, il ne s'agissait en aucune façon de reproduire empiriquement une cabane brésilienne typique au toit de feuilles, et encore moins une hutte traditionnelle indienne (que les dessins, dès les premières illustrations des Aventures de Hans Staden au Brésil, montraient totalement différente, avec une toiture végétale semi-cylindrique). Au lieu de cher-

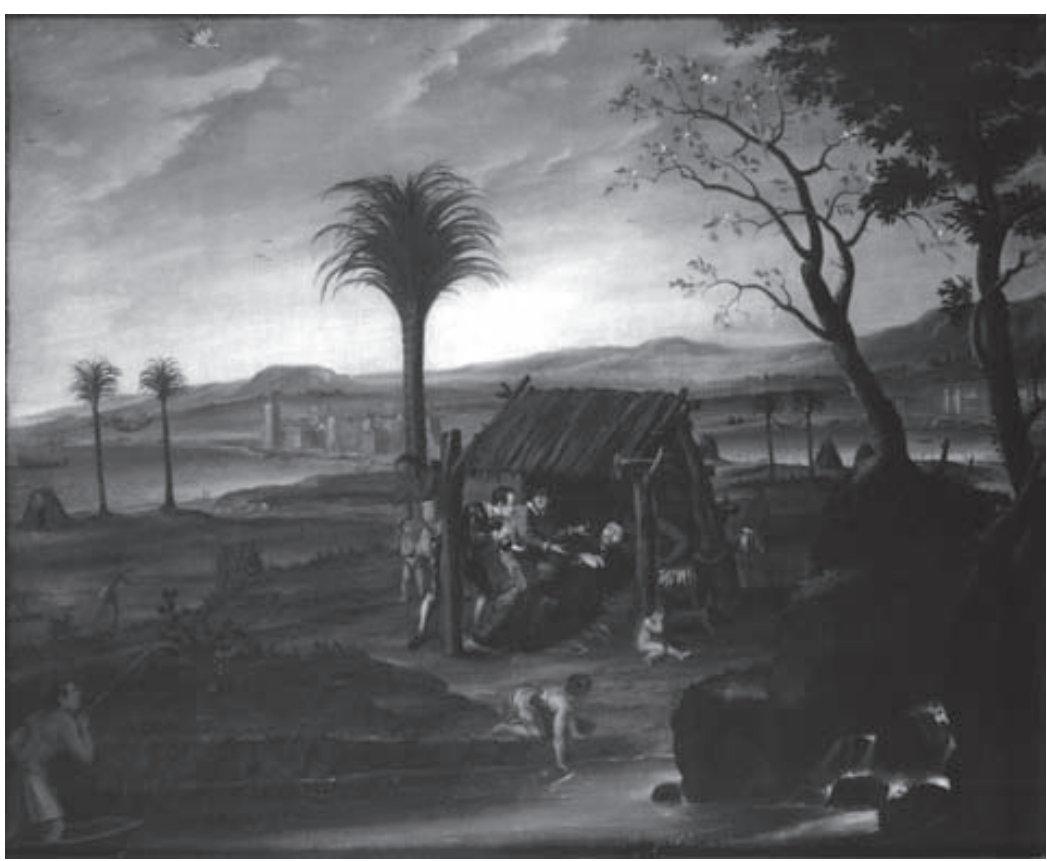
cher une référence locale, I'artiste a procédé par raisonnement humaniste classique : je dois représenter un habitat primitif, donc je cherche I'autorité qui me fournit son modèle. Il n'en existe qu'une, Vitruve. Le prototype de la cabane provient nettement de d'éditions illustrées de De Architectura datant de la Renaissance.

Rien n'a changé au XIXe siècle. Après I'indépendance de 1822 et la constitution de l'empire, il fallait fabriquer un projet historique construire une histoire pour la nouvelle nation - qui s'appuie sur des institutions scientifiques (y compris I'Instituto Histórico e Geográfico Brasileiro créé en 1838), mais aussi sur des formes plus diffuses de la culture. 
À l'époque du romantisme surgit un courant artistique et littéraire dont les conséquences s'étendaient au-delà du champ culturel proprement dit pour déborder, avec force idéologie, sur l'histoire et l'historiographie : I' " indianisme », mouvement qui rendait hommage aux indigènes en leur attribuant noblesse, force hérö̈que et caractère orgueilleux ${ }^{5}$. Cet Indien idéalisé était bien entendu purement imaginaire, et son exaltation culturelle coïncidait dans le temps avec le début de l'extermination des autochtones, qui s'est prolongée jusqu'au XX⿳亠丷厂犬 siècle.

L'Indien fantasmé, devenu un symbole, incarne la noblesse ancestrale revendiquée par les Brésiliens. II permettait aussi de faire abstraction du passé colonial, en offrant une origine autochtone et supérieure. Cette image était présente jusque dans la symbolique officielle : le manteau de couronnement de l'empereur Pedro I était recouvert de plumes de toucan comme si l'empereur recevait cet insigne de pouvoir des habitants ancestraux du pays, effaçant ainsi les siècles de colonisation. Il existe un tableau anonyme, exposé à Tiradentes au Musée Padre Toledo de la fondation Rodrigo Mello Franco de Andrade, qui montre bien le caractère institutionnel de ces procédés allégoriques: devant l'empereur s'agenouille l'Indien, personnification de l'empire brésilien (fig. 3). Le jeune empire autonome renforçait ainsi l'expression de son indépendance vis-à-vis de l'ancienne métropole. Dans les caricatures des journaux du XIXe siècle, l'Indien, souvent présenté comme « Monsieur Brésil », incarnait le pays en tant qu'acteur dans diverses situations politiques.

Cette glorification de l'indigène comme force suprême, entité ancestrale, a eu une incidence sur un autre aspect que nous n'avons pas encore évoqué : elle a fait barrage à la représentation du Noir dans les arts du pays. Les Africains, arrivés au Brésil en tant qu'esclaves, à la différence des Indiens pour qui ce territoire était leur habitat naturel, faisaient partie du quotidien brésilien tant à la campagne qu'en ville. Perçus comme inférieurs, ils étaient en outre la manifestation permanente d'un archaïsme qui se faisait de jour en jour plus insupportable : I'esclavage ne fut aboli qu'en 1888. Grâce à l'Indien, le Noir était mis à l'écart de l'imaginaire collectif et, sauf exception, la littérature et les arts plastiques lui ont accordé peu de place.

Ce sont plutôt les artistes étrangers, et surtout les Français, qui se sont attachés à figurer des types et des comportements de la société brésilienne du XIXe siècle. Après la chute de Napoléon, la cour portugaise, qui avait fui Lisbonne au moment de l'invasion du Portugal par l'armée du général Junot, a prolongé son séjour à Rio de Janeiro, devenue capitale du royaume de Portugal, situation historique connue comme "l'inversion de la métropole ". Très fermé aux étrangers au temps de la colonie, le pays ouvrait alors ses frontières et, en 1816, un groupe d'artistes français conduit par Joachim Lebreton a débarqué à Rio de Janeiro, formant la fameuse Mission artistique française.

Deux de ces peintres ont joué un rôle fondamental dans le portrait du Brésil de cette époque : Nicolas-Antoine Taunay et Jean-Baptiste Debret, formés tous deux par Jacques-Louis David, dont Debret était le cousin. Taunay a repris le thème du paysage, interrompu depuis I'œuvre de Frans Post, et a peint des vues admirables de Rio de Janeiro. Debret, dans son Voyage pittoresque et historique au Brésil, ou Séjour d'un artiste français au Brésil, depuis 1816 jusqu'en 1831 inclusivement $^{6}$ - constitué de 153 planches réalisées dans une visée proprement anthropologique -, relate un quotidien carioca ${ }^{7}$ sans euphémismes et fournit des documents visuels qui montrent la terrible situation des esclaves noirs, victimes de châtiments

3. Anonyme, Alegoria: índio (o Brasil) oferece a coroa ao Imperador Pedro I, sans date, Tiradentes, Museu Padre Toledo/ Fundação Rodrigo Mello Franco de Andrade. 


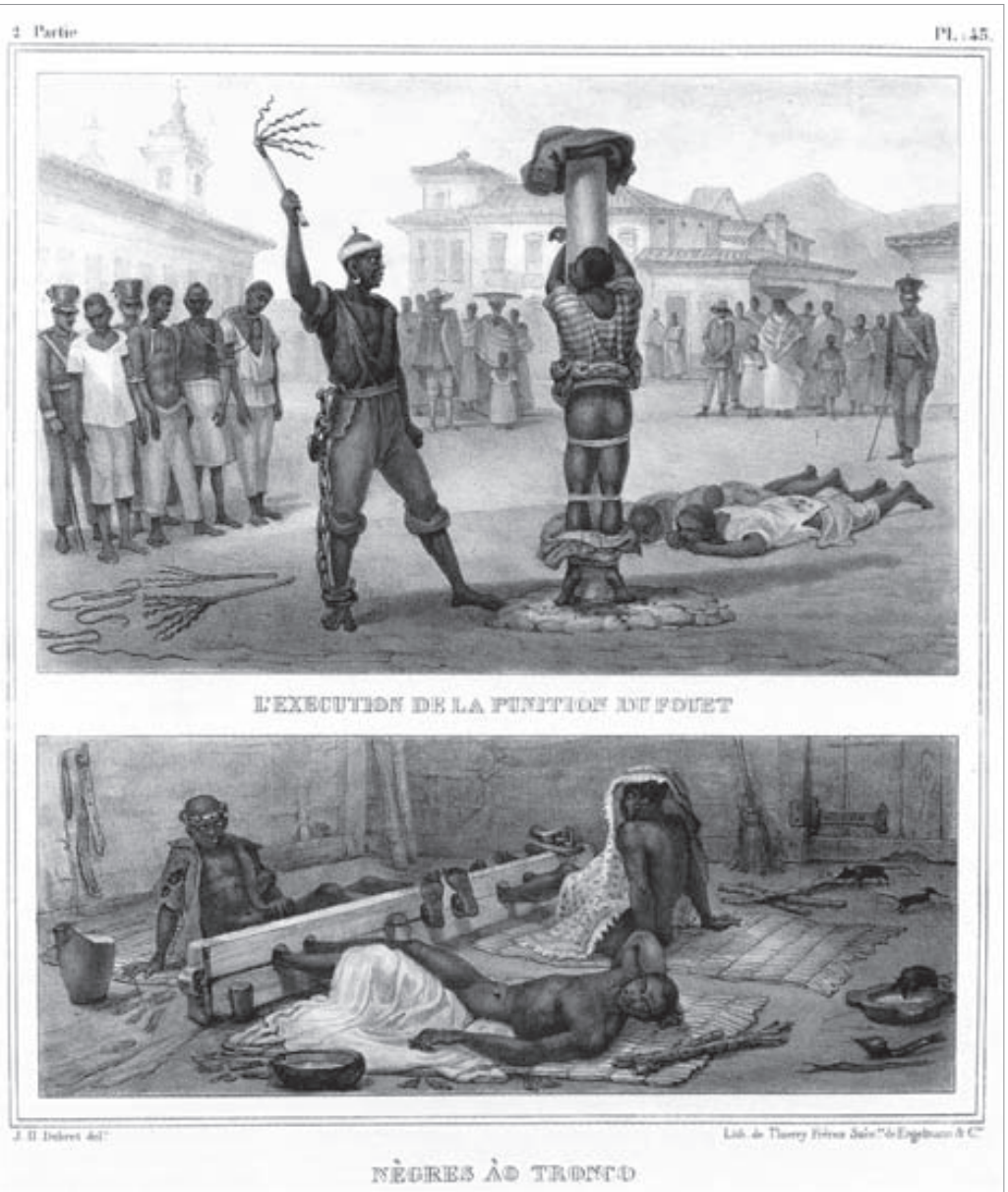

atroces (fig. 4). Comble de l'ironie, ces Français qui ont importé au Brésil les pratiques néoclassiques furent au XIXe siècle perçus par les " modernes " au XXe siècle comme des "ennemis »: on les accusait de falsifier la culture nationale, alléguant que le passé artistique "véritablement » brésilien était le baroque " authentique», comme si le baroque lui-même ne trouvait pas ses racines en Europe...

En fait, ce furent toujours les artistes étrangers qui ont peint la réalité du Brésil, entre autres les Anglais Edwin Landseer et Chamberlain, I'Autrichien Thomas Ender, le Bavarois Johann Moritz Rugendas et le Prussien Ferdinand Theodor Hildebrandt. Tous ont produit des images d'une véritable qualité artistique, mais aussi d'un grand intérêt documentaire. Sans eux, I'histoire n'aurait aucune documentation visuelle du pays, de ses habitants et de leurs comportements pour une bonne partie du XIXe siècle. Pendant ce temps, les artistes brésiliens se consacraient à la noble construction d'une belle histoire, dans laquelle les Indiens, sublimes de caractère et de
4. Jean-Baptiste Debret, Voyage pittoresque et historique au Brésil, ou séjour d'un artiste français au Brésil, depuis 1816 jusqu'en en 1831 inclusivement, Paris, 1835, t. 2, pl. 45 : a. «L'exécution de la punition du fouet »; $\mathbf{b}$. « Nègres ào tronco ". sacrifice, faisaient l'objet de représentations grandioses : Moema de Victor Meirelles, qui ressurgit dans une sculpture importante du même nom de Rodolpho Bernardelli ; Marabá de Rodolpho Amoedo et $O$ último Tamoio du même artiste ; Iracema de José Maria de Medeiros (inspiré du roman homonyme de José de Alencar - notons que Iracema est l'anagramme de «America »; fig. 5) ; la grande sculpture en terre cuite, Alegoria do Império Brasileiro de Chaves Pinheiro, pour ne citer que quelques exemples célèbres ${ }^{8}$. Ce n'est qu'à la fin du siècle qu'Almeida Júnior, peintre de Saõ Paulo, marqué par Gustave Courbet, a prêté attention au personnage du paysan de l'État de São Paulo, construisant son œuvre à partir du vécu et de l'observation.

Au moment de l'exaltation de l'indigène s'est forgé un autre mythe idéologique : celui de la fusion des trois "races ». À l'Indien, resté sans conteste le grand protagoniste ancestral, se sont ajoutés le Portugais et le Noir, dans un métissage harmonieux. Mythe qui fut conforté par le surgissement en 1936 - période de forte idéologie nationaliste - de "I'homme cordial " créé par Sérgio Buarque de Hollanda, qui définit le trait psychologique positif du Brésilien ${ }^{9}$. Cette allégorie fut récemment reprise, en 1995, et avec force, par l'écrivain Darcy Ribeiro dans O povo brasileiro, qui présente une version dramatique de ce métissage sans pourtant démystifier le résultat final, à savoir la fusion des trois matrices de base qui constituent "le Brésilien ${ }^{10}$, une synthèse qui exclut, par présupposé, tous les " étrangers »: 
les nombreux immigrants italiens, japonais, allemands, coréens, etc., arrivés à la fin du XIX ${ }^{\mathrm{e}}$ siècle et qui ont totalement remodelé la géographie humaine du pays au XXe siècle.

Certaines œuvres d'art ont fait beaucoup pour corroborer cette thèse, en particulier deux toiles de Victor Meirelles, A primeira missa no Brasil (1860, Rio de Janeiro, Museu Nacional de Belas Artes; fig. 6) et $A$ batalha dos Guararape (1872-1877, Rio de Janeiro, Museu Nacional de Belas Artes). La première, réalisée à Paris et exposée au Salon de 1861, s'inspira d'un document historique, la Lettre de Pero Vaz de Caminha, dans

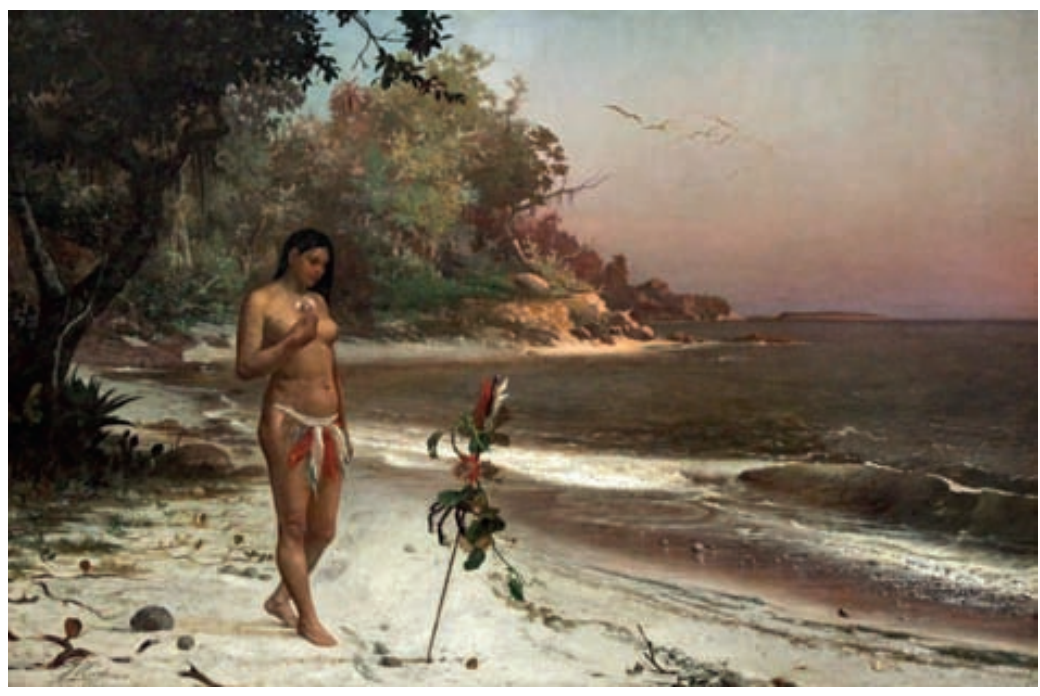
laquelle l'écrivain de bord de la flotte lusitanienne, commandée par Pedro Álvares Cabral, relate au roi du Portugal la découverte du Brésil en 1500. Célébré au XIXe siècle, ce texte, une narration remarquablement vivante publié seulement en $1817^{11}$ - c'est-à-dire au moment où il devenait nécessaire

5. José Maria de Medeiros, Iracema, 1881, Rio de Janeiro, Museu Nacional de Belas Artes d'identifier un document fondateur de la nouvelle histoire -, devint « l'acte de baptême de la Nation ", selon un historien de l'époque. La décision du jury du Salon d'accepter le tableau fut un événement important : pour la première fois, un peintre brésilien, qui avait reçu une bourse du gouvernement impérial, était reconnu par une institution européenne prestigieuse. Dans cette œuvre, Meirelles représente la rencontre pacifique entre les nouveaux venus et les indigènes lors d'une cérémonie chrétienne à ciel ouvert à laquelle participe toute la nature. Le second tableau, $A$ batalha dos Guararape, une immense toile $(494,5 \times 923 \mathrm{~cm})$, relate un combat réunissant en 1654 Portugais, Indiens et Noirs

6. Victor Meirelles, Primeira missa no Brasil, 1860, Rio de Janeiro, Museu Nacional de Belas Artes. contre l'envahisseur hollandais, ultime bataille qui mit fin à l'occupation. Cette union, revendiquée par l'histoire du pays comme la première manifestation d'une " brésilianité » effective, est proclamée en grande pompe par la peinture de Meirelles.

Au début du $X X^{\mathrm{e}}$ siècle, hormis donner une forme nouvelle aux œuvres et une irrévérence amusante aux discours, les « modernes » n'ont pas modifié grand-chose à cette vision : en 1928, I'écrivain moderniste Oswald de Andrade publia son "Manifesto Antropofágico » 12 et Mário de Andrade son roman Macunaíma ${ }^{13}$. Tous deux réitèrent la célébration de l'indigène, et le roman réaffirme le mythe matriciel des «trois races».

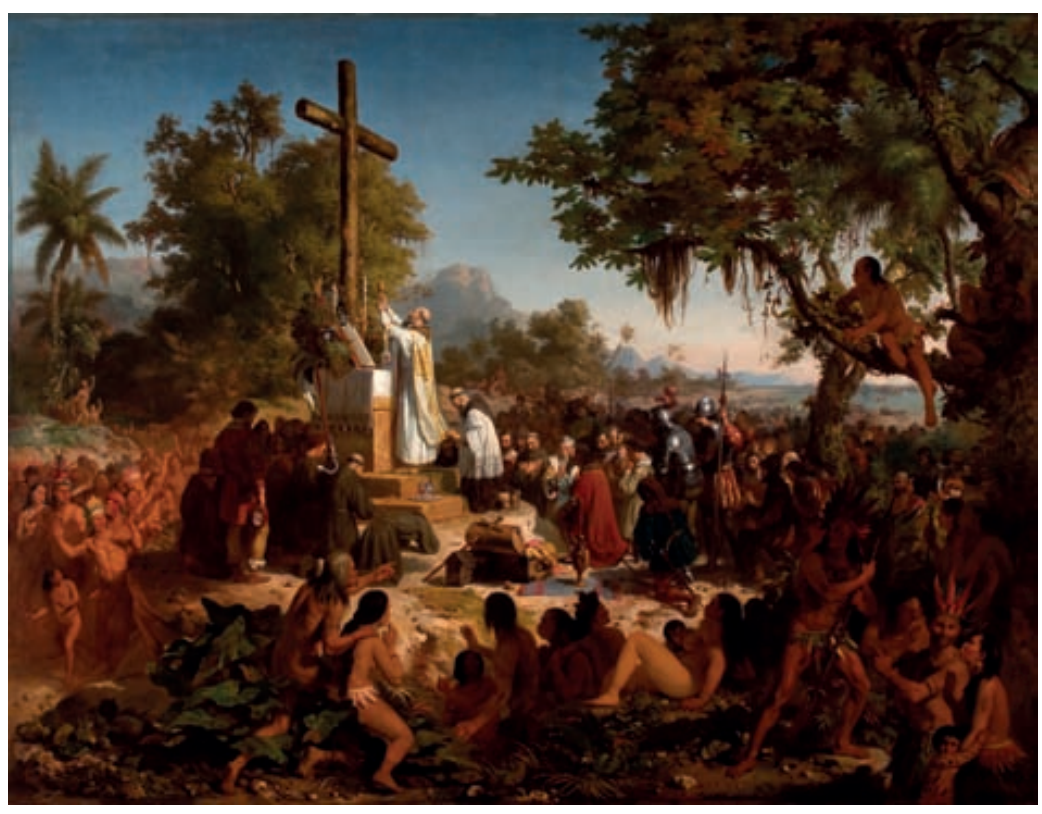




\section{LE BRÉSIL}

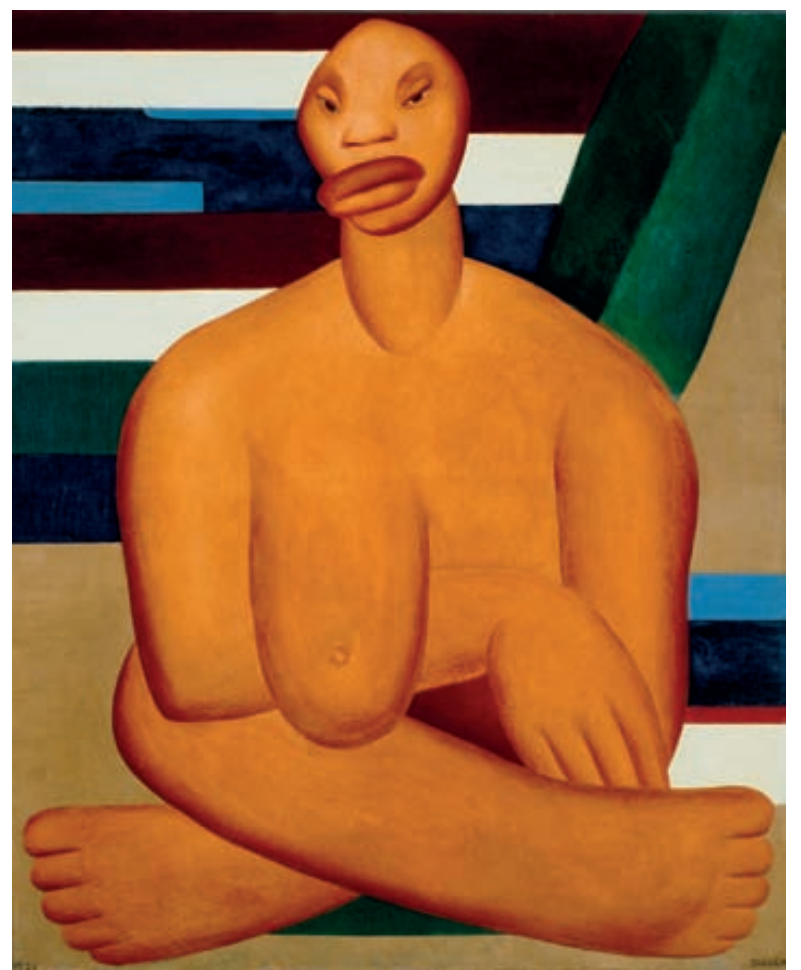

7. Tarsila do Amaral, A Negra, 1923, São Paulo, Museu de Arte Contemporânea da Universidade de São Paulo.
Une œuvre d'exception illustre les procédés modernistes, en ce qu'elle témoigne de la présence africaine dans le pays : A negra, toile de Tarsila do Amaral peinte à Paris en 1923 (fig. 7), fut réalisée en plein apogée de l'art nègre. Ce tableau occupe une place iconique dans l'art brésilien en tant qu'expression authentique d'une négritude nationale. Pourtant, le caractère allégorique de cette image - forte et magnifique au demeurant - qui projette le personnage dans un univers fantastique, exclut bien évidemment une quelconque référence à un réalisme individuel ou social.

Ajoutons que la présence des Noirs dans l'art ne supplante jamais, du moins dans la première moitié du XXe siècle, celle de I'Indien mythique. Le cas d'Emiliano Di Cavalcanti est remarquable. Cet artiste a exploité la mythologie érotique de la mulâtresse - objet de désir sexuel emblématique au Brésil - en lui conférant un traitement d'inspiration " moderne ». Ses célèbres métisses, si nombreuses et si prisées des collectionneurs, constituent un exemple d' " esprit brésilien » dans lequel la récupération de la présence noire se fait en accordant aux belles femmes métissées le rôle d'objet sexuel.

Bien entendu, I'histoire de l'art est partie prenante de cette idéologie nationaliste. À la fin du XIX $X^{e}$ siècle, Gonzaga Duque ${ }^{14}$, la première personnalité marquante de l'histoire de l'art au Brésil, publia Arte Brasileira, un ouvrage essentiel qui dresse un bilan historique de la production artistique du pays, qu'il condamne, avec un parti pris moderne, comme étant conventionnelle, archaïque et dépassée. Sans originalité, écrit-il, ressassant des formules usées, cet art qui naît vieux doit être renouvelé en s'appuyant sur des formes d'expression proprement brésiliennes. Duque affirme avec insistance que le monde artificiel présenté par les artistes est incapable de donner naissance à une école " authentiquement nationale ». Par conséquent, il souhaite l'avènement d'un art qui dépasse les thèmes rebattus et trouve par là une forme à la fois moderne et spécifiquement brésilienne. Son livre a annoncé sans aucun doute les positions modernistes qui ont surgi au début du XXe siècle.

L'histoire culturelle convenue du Brésil signale 1922 comme l'année où le mouvement moderniste voit le jour. Il est sûr que des expériences " modernes » ont eu lieu avant cette date, mais la Semaine d'art moderne, qui s'est déroulée à I'opéra de São Paulo (Theatro Municipal de São Paulo), réunissait les conditions pour choquer une ville de province dont le noyau intellectuel et culturel avait très peu augmenté malgré la croissance de la population, grâce aux immigrations diverses, de 65000 habitants en 1890 à 580000 en 1920. Si les œuvres musicales, littéraires et plastiques présentées n'étaient pas d'un avant-gardisme outrancier, elles étaient suffisamment innovantes pour provoquer un scandale retentissant. C'est au beau milieu de la confusion que s'est affirmée la personnalité marquante de Mário de Andrade, le principal théoricien du mouvement, qui militait pour un art brésilien débarrassé de l'exotisme romantique, prêt à découvrir ses racines profondes et, ainsi, à devenir moderne et actuel (fig. 8). 
Tout comme à l'époque de l'indianisme du XIXe siècle, cette préoccupation nationaliste était le fruit de stimuli venus de l'étranger : les exigences du primitivisme barbare dont les Européens étaient si friands obligeaient les artistes du pays à produire l'art « sauvage » que l'on attendait d'eux, ce qui confortait les Brésiliens dans leur croyance que cette barbarie était leur essence spécifique et naturelle.

Macunaíma, le roman - ou la rapsodie - de Mário de Andrade dont la fiction combine des éléments mythologiques et des traits culturels de toutes les régions du Brésil, fut publié en 1928, en même temps que son Ensaio sobre música brasileira, un livre à caractère théorique qui établit des fondements outrepassant largement le champ musical pour proposer des bases normatives à une pratique générale des arts ${ }^{15}$. Ainsi, avec ces deux ouvrages, l'auteur associait le projet théorique et l'exemple pratique. Ces textes prônent l'idée qu'il faut éliminer tout régionalisme pour favoriser une synthèse (que l'auteur était sans doute convaincu de posséder en lui), sans compter, bien entendu, la tendance propre à tout nationalisme, qui est de gommer les différences de classe au bénéfice d'une identité nationale.

Ces positions étaient prises à l'époque de l'affirmation des pouvoirs totalitaires tant en Europe qu'en Amérique latine. Inutile d'insister sur les affinités entre ces convictions et la dictature des années 1930 de Getúlio Vargas, qui savait enrégimenter les nationalismes d'origines diverses pour les mettre au service de son gouvernement autoritaire. Heitor Villa-Lobos (qui avait participé à la Semaine d'art moderne), après un séjour en Europe où il avait cherché à faire des expériences musicales plus libres et audacieuses, marquées par des traits exotiques attendus par le public européen - à l'époque de ses Choros -, rentra au Brésil et devint le grand compositeur du régime : il créa dans les écoles d'immenses chorales d'enfants et de jeunes, composa des œuvres patriotiques et participa fortement au retour à l'ordre avec ses Bachianas ("à la manière de Bach »), dans lesquelles il mêla l'inspiration du maître de Leipzig à des traits «brésiliens ». Mário de Andrade lui-même fut appelé à Rio de Janeiro à une fonction de conseiller du ministre de l'Éducation, Gustavo Capanema, également chargé des questions culturelles. À son tour, Cândido Portinari, lié à I'histoire du parti communiste, fut invité à concevoir la décoration du siège du ministère de l'Éducation et de la Santé commencée en 1944 et terminée en 1947, dont l'architecture, inspirée du projet de Le Corbusier, fut réalisée par Lúcio Costa, chef d’une équipe d'architectes qui comptait également Oscar Niemeyer. Des sculpteurs de l'envergure de Bruno Giorgi et Jacques Lipchitz, ainsi que le paysagiste Roberto Burle Marx, s'associèrent à cette entreprise, qui officialisa le statut des modernistes.

Les prises de position théoriques nationalistes de Mário de Andrade présupposaient deux ennemis principaux. Le premier était le

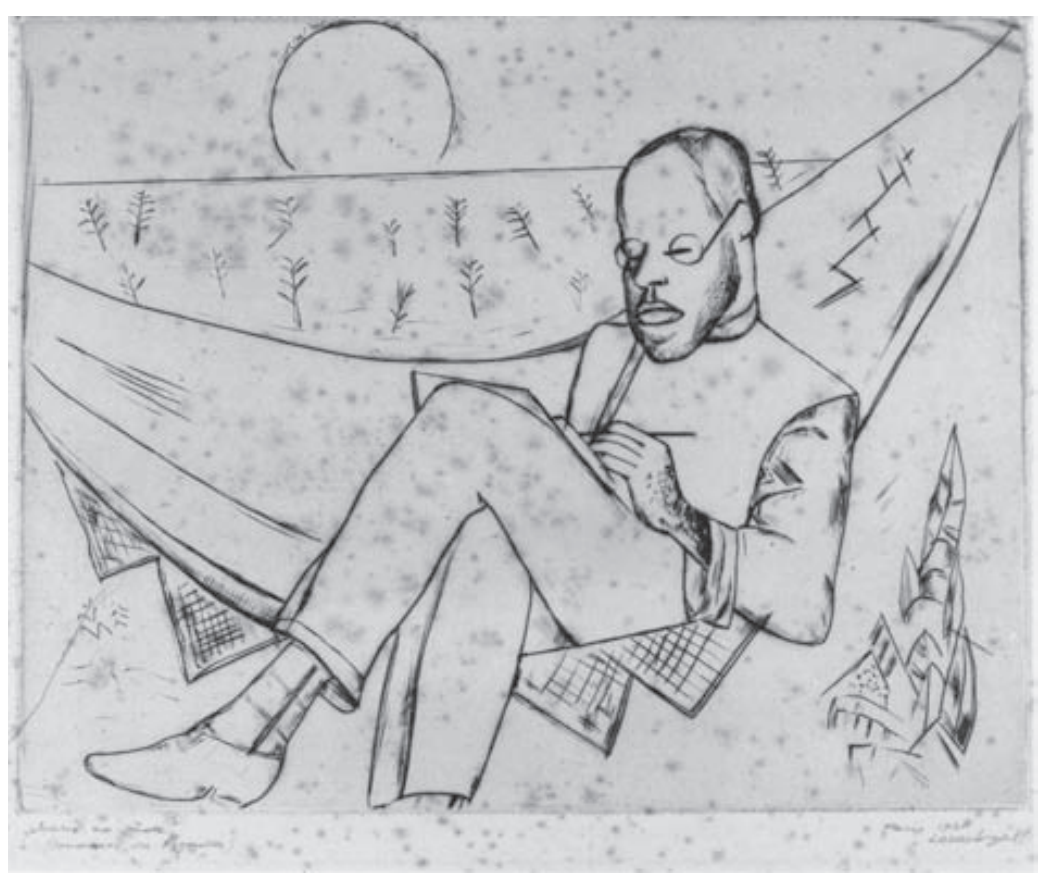

8. Lasar Segall, Mário na rede, 1929, São Paulo, Instituto de Estudos Brasileiros/Universidade de São Paulo, 25,5 x $32 \mathrm{~cm}$, pointe sèche sur papier. 
prestige de la culture internationale, européenne et surtout française, une culture très présente au Brésil : il fallait se protéger des influences indésirables, qui trahissaient ce qu'il voyait comme une « essence » nationale. Le second, plus délicat et d'inflexions sociales plus complexes, était l'affluence des centaines de milliers d'immigrants italiens, japonais, allemands et syro-libanais, entre autres origines, qui, en choisissant de s'établir dans la ville même ou dans l'État de São Paulo, atteignaient directement I'univers géographique de Mário de Andrade et de ses amis modernistes. Le mythe fusionnel et organique des trois « races » servait également à exclure les divers apports non-brésiliens de ces immigrants et à exiger d'eux une intégration à l'idéal national qui impliquait le sacrifice de leurs propres cultures.

Les principes théoriques et idéologiques issus des fondements de cette "culture brésilienne » créée au XIXe siècle ont donc été repris par le mouvement moderniste au XXe siècle. Cette construction, totalement idéologique, fut vécue collectivement - et l'est encore maintenant en grande partie - comme un trait organique, ontogénique. Le mythe des « trois races » était bien utile pour exclure la culture des immigrants, tous affligés du même handicap, celui d'être des travailleurs manuels ou des petits commerçants, au contraire de l'élite locale qui se glorifiait de ses origines luso-indigènes (jamais africaines) sur fond d'aristocratie, inspirant les modernes. C'était aussi un moyen de marteler de façon persistante et répétitive une idéologie sentimentale collective.

C'est dans cet esprit que Mário de Andrade a inventé une histoire de l'art théologique, en procédant à une ingénieuse révision du passé. Elle est déjà constituée dans son Ensaio sobre música brasileira, où il dit vouloir consolider un " esprit de race » et imagine, grosso modo, de diviser la création artistique brésilienne en trois phases, à commencer par la période qu'il appelait « inconsciente ». Au cours de l'histoire, les créateurs se sont laissé pénétrer à leur insu par un esprit brésilien. Le chercheur doit donc s'efforcer d'identifier - par exemple, chez José Maurício, compositeur important de la cour portugaise à Rio de Janeiro - des traits de la brésilianité. Tel est le rôle essentiel de I'historien de l'art : détecter les caractéristiques brésiliennes. Puis vient la période " volontariste » pour employer le terme de Mário de Andrade, à laquelle lui-même et ses contemporains participaient. II fallait « vouloir être » brésilien, se discipliner et s'inspirer de thèmes brésiliens à partir de recherches de préférence dans une perspective anthropologique. Enfin, Mário de Andrade prévoyait un avenir pleinement national : « elle [la musique, et implicitement tous les arts] devra encore s'élever jusqu'à la phase que j'appellerai culturelle, librement esthétique, sans jamais perdre de vue qu'il n'est pas de culture qui ne reflète les réalités profondes du pays où elle est produite. Et alors notre musique sera, non plus nationaliste, mais simplement de chez nous, dans le sens où sont de chez eux un géant tel que Monteverdi ou un mollusque tel que Leoncavallo ${ }^{16}$. Les artistes contemporains devraient donc faire un effort pour être " de chez eux » avant de le devenir «naturellement ».

L'histoire de l'art proposée par Mário de Andrade prétendait chercher des indices "nationaux » dans les œuvres du passé. Il s'agissait donc de découvrir des précurseurs, des artistes qui, la plupart du temps à l'encontre de leur propre intention d'obéir aux modèles européens, avaient intégré dans leurs spécificités et leur style des traits typiques brésiliens; en somme, des agents inconscients de la brésilianité contre les grandes références internationales. Mário de Andrade avait tracé un vecteur dans le temps : plus l'auteur était ancien, plus les marques de brésilianité étaient faibles. À mesure qu'une culture brésilienne s'affirmait progressivement, ces signes se faisaient plus notables. Peu importait la qualité des œuvres. Mário de Andrade redisait avec 
insistance sa méfiance envers le génie : une personnalité créatrice exceptionnelle, avec ses particularismes, nuit au progrès collectif «moyen » de la création artistique. Une «moyenne» qui devient le point crucial de la question : les œuvres significatives qu'une histoire de l'art et de la culture doit prendre en compte ne sont pas les plus innovatrices, les plus élaborées, les plus inventives et extraordinaires. Ce qui compte, c'est le degré de brésilianité, plus ou moins important, plus ou moins précoce ; plus elles échappent aux modèles internationaux, meilleures elles sont.

Cette simplification s'appuyait sur des prémisses à forte teneur idéologique puisqu'il s'agissait de définir ce qui était ou non brésilien, le principe axiomatique le plus profond des particularités nationales.

En mettant en avant les déficiences techniques, ces formulations théoriques ont eu pour conséquence notable de valoriser le caractère rudimentaire, le manque d'habileté des artistes brésiliens dans la pratique de leurs arts respectifs. Des défauts ou des maladresses qui, comparés au savoir-faire et à la dextérité étrangère, pourraient révéler une carence, mais s'analysent, en vérité, comme des manifestations d'une " âme » artistique brésilienne, des nœuds de résistance face à la maîtrise technique montrée dans les centres internationaux. Le goût diffus de l'époque pour un primitivisme généralisé - au plan international - a bien entendu apporté un appui non négligeable à ces partis pris.

L'un des meilleurs instruments de caractérisation nationale a été le folklore, à la fois source d'inspiration artistique et objet de recherche, en premier lieu pour l'érudit polymorphe Mário de Andrade, également ethnologue, anthropologue et folkloriste. L'art moderne brésilien n'a sans aucun doute pas été le seul à se tourner vers le primitivisme et l'archaïsme, mais il faut souligner l'extrême importance que ces notions ont revêtue pour lui.

La théorie évolutionniste de Mário de Andrade s'accompagnait paradoxalement d'une négation de l'histoire, remplacée par ce que l'on pourrait appeler une anthropologie atemporelle, dont on trouve aussi la manifestation dans la pensée de Lúcio Costa, architecte et urbaniste qui s'est lancé dans I'histoire de l'architecture brésilienne, publiant très tôt " O Aleijadinho e a Arquitetura Tradicional » en 192917, puis Documentação necessária ${ }^{18}$ en 1937 et enfin Considerações sobre a arte contemporânea en $1952^{19}$, trois ouvrages dont les interprétations ont marqué et marquent toujours certaines pratiques de déchiffrage des œuvres. Ces textes s'accordent aux vues de Mário de Andrade. Le génie du sculpteur du Minas Gerais connu sous le nom d'Aleijadinho ne participe pas «à l'esprit général de notre architecture », affirme Costa. Pour lui, le point de référence de la brésilianité est l'architecture vernaculaire, domestique, à savoir la maison coloniale. Marcelo Puppi a fait une remarquable analyse dans son Por uma história não moderna da arquitetura brasileira, concluant à ce propos que Costa " prétend instaurer une anthropologie de l'architecture ; ce n'est pas un hasard si celle-ci rend inutile la connaissance effective de l'histoire de sa discipline : I'histoire anthropologique élimine I'histoire historique. La recherche du traditionnel - c'est-à-dire le retour aux racines culturelles de la nation - équivaut à la recherche de notre architecture primitive $»^{20}$. C'est donc le passé colonial, instrument de lutte contre les modes étrangères et délétères du XIXe siècle, qui doit inspirer les architectes du présent.

Ces dogmes ne pouvaient manquer d'avoir des incidences sur la conservation du patrimoine, dont la plus significative est sans doute le maquillage des cités historiques de l'État du Minas Gerais, telles que Tiradentes ou Ouro Preto, où, sous prétexte de préservation, les apports des XIXe et XXe siècles ont été éliminés et où fut imposé auX constructions nouvelles le style du XVIIII siècle. 
S'en suivirent des conséquences également sur les analyses du baroque. La plus importante fut un effet de repli, causée par une recherche axée uniquement sur des processus génétiques et évolutifs internes en refusant les pratiques comparatives avec le reste du monde. Lorsque Germain Bazin séjourna au Brésil en 1945, dans l'élan de la politique culturelle du ministre Capanema, pour développer ses travaux sur l'art et l'architecture baroques, il s'aligna sur les normes nationalistes. La même chose se produisit bien plus tard dans le livre d'Yves Bruand Arquitetura contemporânea no Brasil ${ }^{21}$, premier ouvrage systématique sur le sujet, mais qui adhère aux thèses brésiliennes existantes. Indépendamment des mérites indiscutables de ces auteurs français, leurs horizons demeurent ceux déjà établis par l'idéologie du pays.

Cette idéologie a cependant tremblé dans les années 1950, une période de progrès industriel et de prospérité économique entraînant une ouverture importante sur l'extérieur. Ainsi, le Museu de Arte de São Paulo nouvellement créé a rapidement acquis et exposé une collection de chefs-d'œuvre universels, et la première Bienal Internacional de São Paulo en 1951 a mis les artistes brésiliens en lien avec la production artistique mondiale. Événements marquants mais insuffisants pour s'imposer idéologiquement, et que le coup d'État de 1984 a mis en sourdine, en reprenant à son compte le projet nationaliste.

Pour diverses raisons, l'histoire de l'art au Brésil ne s'est affirmée comme une discipline universitaire solide et autonome que très tard, dans les années 1990. Plusieurs programmes de doctorat furent alors créés, ainsi que, ces dernières années, quelques diplômes spécifiques. Avant cela, les études étaient réalisées par des érudits de formations des plus variées, qui nous ont laissé des ouvrages précieux, mais qui ne possédaient pas l'esprit systématique et critique nécessaire.

Depuis cette évolution capitale, de jeunes historiens de l'art formés selon des principes de rigueur intellectuelle se sont tournés vers un travail exempt de préjugés nationalistes et actualisé au plan international. Beaucoup se sont consacrés à l'étude d'œuvres étrangères, surtout celles qui appartiennent aux collections brésiliennes, mais pas seulement. D'autres se sont passionnés pour des œuvres suscitées par la présence culturelle des immigrants et qui n'avaient pas éveillé l'intérêt jusque-là, par exemple la sculpture monumentale italienne du XXe siècle. Par-dessus tout, l'art brésilien est à présent replacé dans un contexte international, soit par l'étude comparative, soit par le travail sur les archives effectué à l'extérieur du pays sur des artistes brésiliens ayant voyagé. Il est impossible d'énumérer la variété de ces travaux récents qui ont apporté un nouvel élan à l'histoire de l'art du Brésil et fourni de nouveaux éléments de compréhension.

Ce texte a été traduit par Cécile Lombard.

1. Après avoir pris en 1624 la ville de Salvador de Bahia, qu'ils n'ont occupée qu'un an, les Hollandais se sont emparés du Pernambouc en 1630 et ont contrôlé le Nordeste pendant près de vingtquatre ans. Entre 1637 et 1644, lorsqu'il est rentré en Europe, le comte Maurice de Nassau a occupé le poste de gouverneur des territoires conquis et à conquérir par la Compagnie néerlandaise des Indes occidentales au Brésil. Excellent administrateur, il était aussi un humaniste venu dans I'intention de développer un projet scientifique et artistique.
Entouré des érudits et des artistes qu'il avait emmenés, il a créé un musée d'histoire naturelle, un jardin botanique, un jardin zoologique et un observatoire astronomique.

2. Alcídio Mafra Souza éd., O Museu Nacional de Belas Artes, São Paulo, 1985, p. 238.

3. Voir Jorge Coli, "Episódio e alegoria », dans Anuário do Museu Nacional de Belas Artes, v. 1, 2009, p. 105-128.

4. Voir Erwin Panofsky, Problems in Titian: Mostly Iconographic, New York, 1969, p. 28-29. 
5. Les noms les plus importants de la littérature, entre autres, sont, pour la prose, José de Alencar avec ses romans O Guarani (1857), Iracema (1865) et Ubirajara (1874) ; pour la poésie épique, Gonçalves Dias avec I-Juca-Pirama (1851) et Os Timbiras (1856), ainsi que Gonçalves de Magalhães avec A confederação dos Tamoios (1856). En peinture, un peu plus tardivement, on trouve Victor Meirelles, Rodolfo Amoedo et Antônio Parreiras. Une place importante doit être réservée au compositeur Antonio Carlos Gomes, qui a représenté à la Scala de Milan I'opéra // Guarany en 1870, tiré du roman d'Alencar, et créé Lo Schiavo à Rio de Janeiro en 1889

6. Jean-Baptiste Debret, Voyage pittoresque et historique au Brésil, ou Séjour d'un artiste français au Brésil, depuis 1816 jusqu'en 1831 inclusivement, Paris, 1834

7. Adjectif employé pour faire référence à Rio de Janeiro.

8. Victor Meirelles, Moema (1866, São Paulo, Museu de Arte de São Paulo), inspiré du poème épique de Santa Rita Durão, Caramuru (1781) ; Rodolpho Bernardelli, Moema (1895, Pinacoteca do Estado de São Paulo) ; Rodolpho Amoedo, Marabá (1882, Rio de Janeiro, Museu Nacional de Belas Artes), inspiré du poème homonyme de Gonçalves Dias (1851); Rodolpho Amoedo, O último Tamoio (1883, Rio de Janeiro, Museu Nacional de Belas Artes), inspiré du poème épique de Gonçalves de Magalhães A confederação dos Tamoios (1856) ; José Maria de Medeiros, Iracema (1884, Rio de Janeiro, Museu Nacional de Belas Artes), inspiré du roman homonyme de José de Alencar (1865); Chaves Pinheiro, Alegoria do Império Brasileiro (1871, Rio de Janeiro, Museu Nacional de Belas Artes).

9. Sérgio Buarque de Holanda, Raízes do Brasil, São Paulo, 1936.

10. Darcy Ribeiro, O povo brasileiro: a formação e o sentido do Brasil, São Paulo, 1995.

11. La Lettre de Pero Vaz de Caminha fut publiée dans Corografia Brasílica de Manuel Aires de Casal (Rio de Janeiro, 1817).

12. Oswald de Andrade (1890-1954), poète, romancier, essayiste et polémiste, fut l'un des précurseurs du mouvement moderniste et l'un des créateurs et participants de la Semaine d'art moderne de São Paulo en 1922. Son "Manifesto Antropófago ", publié en mai 1928 dans le premier numéro de la revue Revista de Antropofagia dont il fut le fondateur, exprimait I'idée que le cannibalisme culturel, naturellement inspiré de pratiques indigènes - absorption de toutes les formes de culture internationale pour les digérer et les transformer en culture brésilienne - était un moyen d'affirmer une culture nationale contre l'Europe.

13. Mário de Andrade (1893-1945), poète, romancier, musicologue, professeur au conservatoire de São Paulo, critique et historien de l'art, et principal théoricien du mouvement moderniste, a lui aussi joué un rôle essentiel dans la réalisation de la Semaine d'art moderne. Son prestige en tant qu'érudit était immense, et ses interventions parmi les artistes brésiliens de tous les bords ont été déterminantes. Macunaíma (São Paulo, 1928) est un roman - que I'auteur appelle « rapsodie »-dans lequel par des situations irréelles, fondées en grande partie sur la mythologie indienne, il cherche à offrir une synthèse de la fameuse brésilianité

14. Luiz Gonzaga Duque Estrada fut critique d'art à Rio de Janeiro ; il fut l'auteur de $A$ arte brasileira: pintura e esculptura (Rio de Janeiro, 1888) et, outre d'autres textes consacrés à l'art, du roman à clé $M o$ cidade morta (Rio de Janeiro, 1899), dans lequel il dresse un portrait du milieu artistique carioca de la fin du XIXe siècle.

15. Andrade, 1928, cité n. 12 ; Mário de Andrade, Ensaio sobre música brasileira, São Paulo, (1928) 1962.

16. « [...] ela terá que se elevar ainda à fase que chamarei de Cultural, livremente estética, e sempre se entendendo que não poderá haver cultura que não reflita as realidades profundas da terra em que se realiza. E então a nossa música será, não mais nacionalista, mas simplesmente nacional, no sentido em que são nacionais um gigante como Monteverdi e um molusco como Leoncavallo » (Andrade, [1928] 1962, cité n. 15, p. 33-34). Ce schéma fut repris en 1941, dans une perspective historique plus accentuée, dans l'étude «Evolução Social da Música no Brasil », éditée postérieurement dans le volume $A s$ pectos da música brasileira des œuvres complètes de Mário de Andrade (São Paulo, 1965).

17. Lúcio Costa, "O Aleijadinho e a arquitetura tradicional ", dans Sobre arquitetura, Porto Alegre, 1966, p. 12-16 [éd. orig. dans O Jornal, 1929].

18. Lúcio Costa, "Documentação necessária ", dans Costa, 1966, cité n. 17, p. 202-229 [éd. orig. dans Revista do SPHAN, 1, 1937].

19. Costa, 1966, cité n. 16, p. 202-229 [éd. orig. : Considerações sobre a arte contemporânea, Rio de Janeiro, 1952].

20. «[...] pretende instaurar uma antropologia da arquitetura; esta não por acaso torna desnecessário o conhecimento efetivo da própria história disciplinar: a 'história' antropológica elimina a história 'histórica'. A procura do tradicional - vale dizer, o retorno às raízes culturais da nação - equivale à busca de nossa arquitetura primitiva » (Marcelo Puppi, Por uma história não moderna da arquitetura brasileira, Campinas, 1998).

21. Yves Bruand, Arquitetura contemporânea no Brasil, Campinas, 1981. 